\title{
Evaluation of cartridge-based nucleic acid amplification test for diagnosis of tuberculosis in children in various body fluids
}

\author{
Dipti Agarwal', Rakesh Bhatia ${ }^{2}$, Rajeshwar Dayal ${ }^{3}$, Rhythm Khera ${ }^{4}$, Shamrendra Narayan ${ }^{5}$, Ankur Goyal $^{6}$ \\ From ${ }^{1}$ Associate Professor, Department of Pediatrics, ${ }^{5}$ Associate Professor, Department of Radiodiagnosis, Dr. RMLIMS, Lucknow, ${ }^{2}$ Professor, \\ ${ }^{3}$ Professor, ${ }^{4}$ Resident, Department of Pediatrics, ${ }^{6}$ Associate Professor, Department of Microbiology, SNMC, Agra, Uttar Pradesh, India
}

Correspondence to: Dr. Shamrendra Narayan, Flat 406; Faculty Apartment, TC40-41/V Vibhuti Khand, Lucknow - 226010 , Uttar Pradesh, India. E-mail: samarnarayan@yahoo.co.in

Received - 18 May 2019

Initial Review - 10 June 2019

Accepted - 17 June 2019

\begin{abstract}
Introduction: The paucibacillary nature presents a major challenge in the diagnosis of tuberculosis (TB) in children. The utilization of cartridge-based nucleic acid amplification test (CBNAAT) for the diagnosis of TB presents itself with added advantages such as detection to resistance to rifampicin and short turnaround time. Objectives: The aim of the study is to evaluate the diagnostic yield of CBNAAT in various body fluids and to compare with BACTEC-MGIT 960 and acid-fast bacilli (AFB) microscopy in children with suspected TB and to see the prevalence of rifampicin resistance in the study population using CBNAAT. Materials and Methods: This cross-sectional study included participants $<14$ years with suspected TB. Gastric aspirate samples obtained from pulmonary TB cases and body fluid specimens obtained from extrapulmonary TB cases were processed for the detection of Mycobacterium tuberculosis (MTB) using CBNAAT, BACTEC-MGIT 960, and AFB microscopy. The results obtained using CBNAAT were compared to other laboratory tests using an appropriate statistical method. Results: Fifty patients diagnosed with TB (34 pulmonary, 10 pleural effusion, and 6 abdominal) were included in the study, and clinical fluid specimens obtained from study participants were processed for the detection of MTB. Out of 34 gastric aspirate samples, $28(82 \%)$ were positive by CBNAAT which was statistically higher than BACTEC-MGIT $960(P<0.05)$. Among extrapulmonary TB cases, only 2 pleural fluid specimens were positive by CBNAAT, whereas BACTEC-MGIT 960 and AFB microscopy could not detect MTB. Out of 34, 4 (11.76\%) patients with newly diagnosed pulmonary TB were found to be rifampicin resistant using CBNAAT. Conclusions: CBNAAT showed promising results as a diagnostic tool in detecting MTB and rifampicin resistance in pulmonary TB using gastric aspirate. It, however, did not show good results in children with extrapulmonary TB in the clinical fluid specimen. The present study also showed the presence of high rifampicin resistance in treatment naïve pulmonary TB patients.
\end{abstract}

Key words: Acid-fast bacilli microscopy, BACTEC 960, Cartridge-based nucleic acid amplification test, Children, Diagnosis, Tuberculosis

$\mathrm{T}$ uberculosis (TB) is one of the leading causes of death from infectious disease worldwide. Globally, 10.4 million of new cases of TB disease were reported in the year 2015 by the WHO, with an estimated 1 million children $<14$ years of age [1]. Rapid emergence of drug resistance in recent years is another major issue in the management of TB. Globally, an estimated $3.6 \%$ of new TB cases and $20 \%$ of previously treated cases have multidrug-resistant TB [1]. Diagnosis of TB in children is challenging because of paucibacillary nature. The drawback of the conventional culture method is that it is positive in $<50 \%$ of cases and is time-consuming [2].

BACTEC-MGIT 960 culture system allows rapid detection of mycobacteria and has reported a sensitivity of $51.5 \%$ and $48.6 \%$ in pulmonary and extrapulmonary TB participants, respectively [3,4]. Cartridge-based nucleic acid amplification test (CBNAAT) needs only 150 bacilli per $\mathrm{ml}$ of clinical specimen to be positive [5]. Meta-analysis study conducted showed that pooled sensitivity of CBNAAT was observed to be $78 \%$ in gastric aspirate samples, $34 \%$ in pleural fluid, and $<1 \%$ for non-pleural fluid samples [6]. CBNAAT can simultaneously detect resistance to rifampicin and has the advantage of short turnaround time [7].

Hence, this study was undertaken to assess the diagnostic yield of CBNAAT in comparison to BACTEC-MGIT 960 and Zeihl-Neelson microscopy in gastric aspirate specimens of pulmonary $\mathrm{TB}$ cases and in specimens obtained from serous cavities in extrapulmonary TB cases. Detection of resistance to rifampicin was also assessed in these participants using CBNAAT.

\section{MATERIALS AND METHODS}

A cross-sectional study was conducted in children up to 18 years of age from the period of March 2015 to June 2017. The inclusion criteria consisted of participants with suspected TB attending the 
Department of Pediatrics of tertiary care hospital during the study period. The cases were diagnosed in accordance to the National Guidelines on the Diagnosis of Pediatric [8] TB. Participants with pulmonary TB and extrapulmonary TB with serous cavity involvement were included in the study. Patients with a history of receiving antitubercular therapy and with central nervous system TB were excluded from the study. Ethical clearance was obtained from the institutional ethical committee. Informed consent was obtained from the caretaker/guardian of the participants enrolled in the study.

A detailed history and thorough clinical examination were done in all the study participants. Chest X-ray and tuberculin skin test were also performed in all the participants. Five milliliter of serous fluid sample (pleural/ascitic/pericardial fluid) from extrapulmonary TB cases and $5 \mathrm{ml}$ of gastric aspirate samples were obtained from pulmonary TB cases under aseptic conditions. These samples were transported to laboratory maintaining the cold chain on the same day for the following investigations: cytological and biochemical tests, acid-fast bacilli (AFB) microscopy, culture on BACTEC-MGIT 960, and CBNAAT.

Data were collected and analyzed using SPSS software version 20. The sensitivity of CBNAAT, BACTEC-MGIT 960, and AFB microscopy was calculated. McNemar's Chi-square test was applied to compare the sensitivity CBNAAT with BACTEC-MGIT 960 and AFB microscopy. A $\mathrm{p} \leq 0.05$ was taken as statistically significant. Taking BACTEC-MGIT 960 as gold standard, specificity, sensitivity, positive predictive value (PPV), negative predictive value (NPV), negative likelihood ratio, and positive likelihood ratio of CBNAAT were calculated.

\section{RESULTS}

A total of 50 patients with diagnosed TB cases were registered during the study period: 34 were pulmonary TB, 10 were pleural effusions, and 6 were abdominal TB. The clinical specimen was obtained from these patients ( 34 gastric aspirate, 10 pleural fluid, and 6 ascitic fluid).

Fever was the most common clinical presentation in the study participants $(80 \%$ case). According to the WHO classification, $80 \%$ of cases were malnourished; $36 \%$ were moderately; and $44 \%$ were severely malnourished. Clinical and demographic profile of the study participants is depicted in Table 1. Chest X-ray was abnormal in $32(94 \%)$ of 34 pulmonary TB cases, showing hilar lymphadenopathy in 26 cases (76\%), pulmonary infiltrates in 12 cases $(24 \%)$, consolidation in 5 cases $(10 \%)$, and military shadow in 4 cases $(8 \%)$. Out of 6 cases of abdominal TB, only $2(33.3 \%)$ showed hilar lymphadenopathy in chest X-ray.

The yield of CBNAAT, BACTEC-MGIT 960, and AFB smear microscopy for gastric aspirate, pleural, and ascitic fluid samples is shown in Table 2. On comparing CBNAAT with AFB smear microscopy and BACTEC-MGIT 960 in gastric aspirate samples, there was statistically higher yield $(P=0.000003$ and 0.00814, respectively). Taking BACTEC-MGIT 960 as the gold standard, sensitivity of CBNAAT was 100\% (95\%
Table 1: Clinical and demographic profile of tuberculosis suspect cases $(\mathbf{n}=\mathbf{5 0})$

\begin{tabular}{lc}
\hline Variables & $\mathbf{n}(\mathbf{\%})$ \\
\hline Demographic & \\
Mean age & $4.97 \pm 2.82$ \\
Male gender & $29(58)$ \\
History of contact & $29(58)$ \\
BCG vaccination & $23(46)$ \\
Mantoux positive & $28(56)$ \\
Clinical & \\
Persistent fever & $40(80)$ \\
Cough >2 weeks & $36(72)$ \\
Loss of weight/no weight gain & $27(54)$ \\
Anorexia & $19(38)$ \\
Respiratory distress & $18(36)$ \\
Abdomen distension & $6(12)$ \\
Pain abdomen & $3(6)$ \\
Diarrhea/constipation & $4(8)$ \\
\hline
\end{tabular}

BCG: Bacillus Calmette-Guérin

confidence interval [CI]: 83.89-100\%) and specificity was 46.15\% (95\% CI: $19.22-74.87 \%$ ), positive likelihood ratio was 1.86 (95\% CI: $1.12-3.07 \%$ ), negative likelihood ratio was 0, PPV was $75 \%$ (95\% CI: $55.13-89.31 \%)$, and NPV was $100 \%(95 \%$ CI: $54.07-100 \%)$.

CBNAAT detected Mycobacterium tuberculosis in $2(20 \%)$ specimens of pleural fluid but could not detect in any of the ascitic fluid specimens. BACTEC-MGIT 960 and microscopy could not detect MTB in pleural and ascitic fluid specimens. There was no significant difference seen in comparing CBNAAT with other diagnostic tests for body fluid samples. Out of 34 pulmonary TB cases, $4(11.76 \%)$ were found to have rifampicin resistance and no resistance was detected among extrapulmonary cases.

\section{DISCUSSION}

CBNAAT has been endorsed by the WHO to be used for an initial diagnostic test in children suspected of having pulmonary as well as extrapulmonary TB $[7,8]$. In the present study, sensitivity of CBNAAT was assessed in gastric aspirate, pleural fluid, and ascitic fluid specimens obtained from pulmonary, pleural, and extrapulmonary TB participants, respectively. In the present study, the mean age of the study participants was $4.97 \pm 2.82$ years. A similar observation has been made in a study by Anane and Grangaud, showing that the risk of acquiring TB was higher in younger children [9]. Male predominance was seen in the present study as also reported by Jani et al. [10].

Fever was the most common clinical presentation followed by loss of weight, decreased appetite which was similar to the observations made by Anane and Grangaud [9]. Tuberculin test was found to be positive in about $50 \%$ participants in our study. Mehta et al. also found positive tuberculin testing in $40 \%$ of children with TB [11]. Lower tuberculin positivity could be due to coexistent undernutrition in the study participants. In our study, a 
Table 2: Comparison of cartridge-based nucleic acid amplification test, BACTEC-MGIT 960, and acid-fast bacilli microscopy in various body fluids

\begin{tabular}{lccc}
\hline Clinical specimen, $n(\%)$ & CBNAAT positive & BACTEC-MGIT 960 positive & AFB microscopy positive \\
\hline GA $(n=34)$ & $28(82)$ & $21(67.7)$ & $6(18)$ \\
PF $(n=10)$ & $2(20)$ & 0 & 0 \\
AF $(n=6)$ & 0 & 0 & 0 \\
Total $(n=50)$ & $30(60)$ & $21(42)$ & $6(12)$ \\
\hline
\end{tabular}

GA: Gastric aspirate, PF: Pleural fluid, AF: Ascitic fluid, CBNAAT: Cartridge-based nucleic acid amplification test, AFB: Acid-fast bacilli

large number of patients was malnourished; a similar observation was made in a study by Jaganath and Mupere which showed high TB prevalence in severely malnourished children [12].

In the present study, the yield of microscopy, BACTEC-MGIT 960, and CBNAAT was compared in gastric aspirate samples. In the present study, smear microscopy was positive in the small number of gastric aspirate samples. In a study conducted by Bates et al., smear microscopy was positive in $25 \%$ of gastric aspirate samples [13]. BACTEC-MGIT 960 was positive in more than half participants who were higher than the study conducted by Ruiz Jiménez et al. who showed culture to be positive in $47.1 \%$ in gastric aspirates [14]. In our study, BACTEC-MGIT 960 performed slightly better than the previous studies probably due to a better sample collection technique.

Sensitivity of CBNAAT was $82 \%$ which was higher than the sensitivity reported by Bates et al. which was $68.8 \%$ (95\% CI: 53.6-80.9\%) [13]. In a meta-analysis conducted by Maynard-Smith et al., pooled sensitivity estimate of CBNAAT in gastric aspirate was 78\% (95\% CI: 69-86\%) [6]. In the present study, CBNAAT when compared with BACTECMGIT 960 taken as reference test showed 100\% sensitivity. In another study conducted by Pang et al., CBNAAT compared with BACTEC-MGIT 960 showed sensitivity of $64.7 \%$ and specificity of $70.1 \%$ [15]. Hence, CBNAAT can be used as an initial diagnostic test for diagnosing pulmonary TB using gastric aspirate samples.

Extrapulmonary TB cases were evaluated in the study using various diagnostic tools. BACTEC-MGIT 960 and microscopy were negative in pleural and ascitic fluid samples. In a study conducted by Chakravorty et al., smear microscopy in extrapulmonary TB showed positivity of 3.9\% [16]. Zmak et al. found only 1 out of 42 pleural fluid and 3 out of 10 ascitic fluid to be culture positive [17]. Low sensitivity in the present study was probably due to paucibacillary nature of pleural and ascitic fluid coupled with a small sample size of the study group. In a study conducted by Friedrich et al., sensitivity of CBNAAT assay in pleural fluid was $25 \%$ [18]. In a meta-analysis conducted by Maynard-Smith et al., pooled sensitivity estimate of CBNAAT in pleural fluid was 34\% (95\% CI: $24-44 \%)$ and for non-pleural serous fluid was $<1 \%$ [6].

In the present study, rifampicin resistance was detected at an alarmingly high rate in new cases of pulmonary TB cases using CBNAAT as also observed in another study by Sharma et al. [19]. A meta-analysis by Chang et al. has shown effectiveness and rapidity in diagnosis of rifampicin resistance [20]. The major limitation that the present study faced was the small number of patients in the study population. Hence, to overcome the outcome from this limitation, further studies should be designed with larger number of participants in the study population.

\section{CONCLUSIONS}

CBNAAT showed promising results for the diagnosis of pulmonary TB using gastric aspirate in children. It had higher sensitivity compared to currently available diagnostic modalities including smear microscopy and BACTEC-MGIT 960. CBNAAT, however, does not show good results in children with extrapulmonary TB in pleural and ascitic fluid. The study also shows high rifampicin resistance in new cases of pulmonary TB. To conclude, CBNAAT may be a promising diagnostic test as compared to conventional methods for an initial diagnostic test in children with suspected pulmonary and extrapulmonary TB and to detect rifampicin resistance.

\section{REFERENCES}

1. World Health Organization. Global Tuberculosis Report. World Health Organization 2018. Available from: http://www. apps.who.int. [Last accessed on 2018 Dec 25].

2. Yeager H Jr., Lacy J, Smith LR, LeMaistre CA. Quantitative studies of mycobacterial populations in sputum and saliva. Am Rev Respir Dis 1967;95:998-1004.

3. Palacios JJ, Ferro J, Ruiz Palma N, García JM, Villar H, Rodríguez J, et al. Fully automated liquid culture system compared with löwenstein-jensen solid medium for rapid recovery of mycobacteria from clinical samples. Eur J Clin Microbiol Infect Dis 1999;18:265-73.

4. Negi SS, Khan SF, Gupta S, Pasha ST, Khare S, Lal S, et al. Comparison of the conventional diagnostic modalities, bactec culture and polymerase chain reaction test for diagnosis of tuberculosis. Indian J Med Microbiol 2005;23:29-33.

5. GeneXpert Brochure-National Health Laboratory Service. Available from: http://www.nhls.ac.za/assets/files/GeneXpert\%20brochure.pdf. [Last accessed on 2019 Jan 13].

6. Maynard-Smith L, Larke N, Peters JA, Lawn SD. Diagnostic accuracy of the xpert MTB/RIF assay for extrapulmonary and pulmonary tuberculosis when testing non-respiratory samples: A systematic review. BMC Infect Dis 2014;14:709.

7. World Health Organization. Xpert MTB/RIF: World Health Organization Policy Update and Implementation Manual. Available from: https://www. who.int.xpert. [Last accessed on 2018 Dec 23].

8. Guidelines on Pediatric TB Central TB Division. Available from: http:// www.tbcindia.nic.gov.in. [Last accessed on 2018 Dec 26].

9. Anane T, Grangaud JP. Diagnosis of tuberculosis in children. Child Trop 1992;196-197:20-9.

10. Jani Y, Sarvaiya AN, Thakor N. Socio demographic profile of pediatric tuberculosis patients of north Gujarat region, India: A cross sectional study. Int J Res Med Sci 2015;3:3382-5.

11. Mehta R, Saini L, Mittal SK. A critical evaluation of B.C.G. Test applicability in pediatric practice. Indian Pediatr 1986;23:419-28. 
12. Jaganath D, Mupere E. Childhood tuberculosis and malnutrition. J Infect Dis 2012;206:1809-15.

13. Bates M, O'Grady J, Maeurer M, Tembo J, Chilukutu L, Chabala C, et al. Assessment of the xpert MTB/RIF assay for diagnosis of tuberculosis with gastric lavage aspirates in children in Sub-Saharan Africa: A prospective descriptive study. Lancet Infect Dis 2013;13:36-42.

14. Ruiz Jiménez M, Guillén Martín S, Prieto Tato LM, Cacho Calvo JB, Álvarez García A, Soto Sánchez B, et al. Induced sputum versus gastric lavage for the diagnosis of pulmonary tuberculosis in children. BMC Infect Dis 2013;13:222.

15. Pang Y, Wang Y, Zhao S, Liu J, Zhao Y, Li H, et al. Evaluation of the xpert MTB/RIF assay in gastric lavage aspirates for diagnosis of smear-negative childhood pulmonary tuberculosis. Pediatr Infect Dis J 2014;33:1047-51.

16. Chakravorty S, Sen MK, Tyagi JS. Diagnosis of extrapulmonary tuberculosis by smear, culture, and PCR using universal sample processing technology. J Clin Microbiol 2005;43:4357-62.

17. Zmak L, Jankovic M, Jankovic VK. Evaluation of xpert MTB/RIF assay for rapid molecular diagnosis of tuberculosis in a two-year period in Croatia. Int J Mycobacteriol 2013;2:179-82.
18. Friedrich SO, von Groote-Bidlingmaier F, Diacon AH. Xpert MTB/RIF assay for diagnosis of pleural tuberculosis. J Clin Microbiol 2011;49:4341-2.

19. Sharma SK, Kohli M, Yadav RN, Chaubey J, Bhasin D, Sreenivas V, et al. Evaluating the diagnostic accuracy of xpert MTB/RIF assay in pulmonary tuberculosis. PLoS One 2015;10:e0141011.

20. Chang K, Lu W, Wang J, Zhang K, Jia S, Li F, et al. Rapid and effective diagnosis of tuberculosis and rifampicin resistance with xpert MTB/RIF assay: A meta-analysis. J Infect 2012;64:580-8.

Funding: None; Conflict of Interest: None Stated.

How to cite this article: Agarwal D, Bhatia R, Dayal R, Khera R, Narayan S, Goyal A. Evaluation of cartridge-based nucleic acid amplification test for diagnosis of tuberculosis in children in various body fluids. Indian J Child Health. 2019; 6(7):349-352.

Doi: 10.32677/IJCH.2019.v06.i07.005 